\title{
Correction of malposition of
} type diagnostic $\mathrm{X}$-ray apparatus

\section{-a case report-}

\author{
Jaekyu Ryu, Ji-Hyun Yoon, Eun-Joon Lee, Chia An Lee, Seong Chang Woo, \\ and Chang-Young Jeong
}

Department of Anesthesioloy and Pain Medicine, Eulji University School of Medicine, Daejeon, Korea

Central venous catheters provide long-term available vascular access. They are useful for central venous pressure monitoring, rapid fluid management, massive transfusion and direct cardiovascular medication, especially in operation. Central venous catheterization is usually performed by the landmark bedside technique without imaging guidance. The complications of central venous catheterization are frequent, which include malposition, pneumothorax, hemothorax, chylothorax, arterial puncture, hematoma, air embolism and infection. Malposition of a central venous catheter is not rare and may cause several complications such as malfunction of the catheter, default measurement of central venous pressure, catheter erosion, thrombophlebitis and cardiac tamponade. In this case, we report a malposition of central venous catheter with 9-Fr introducer sheath which is located in the right subclavian vein via ipsilateral internal jugular vein and the correction of this misplacement assisted by mobile type diagnostic X-ray apparatus (C-arm fluoroscope).

Key Words: Catheterization, Internal jugular vein, Malposition, Subclavian vein.

A central venous catheter may be used for various purposes, including rapid infusion (of drugs, solution, or blood), total parenteral nutrition, and monitoring of central venous pressure. A central venous catheter is usually inserted by venipuncture at appropriate locations, such as the subclavian vein, internal jugular vein, and femoral vein, while following surface ana-

Corresponding author: Chang-Young Jeong, M.D., Ph.D.

Department of Anesthesioloy and Pain Medicine, Eulji University School of Medicine, 95, Dunsanse-ro, Seo-gu, Daejeon 302-799, Korea

Tel: 82-42-611-3883, Fax: 82-42-259-1111

E-mail: cyjeong@jnu.ac.kr

Received: February 19, 2014.

Revised: 1st, April 8, 2014; 2nd, April 15, 2014.

Accepted: April 17, 2014.

Korean J Anesthesiol 2015 August 68(4): 402-406

http://dx.doi.org/10.4097/kjae.2015.68.4.402 tomical landmarks without particular image guidance during operations, treatment, or emergency room care. Therefore, even experienced physicians are not free from complication risks, which include pneumothorax, hemothorax, chylothórax, arteriopuncture, hematoma, damage to nerve tissue or surrounding tissue, arrhythmia, thrombophlebitis, and abnormal catheter position. In addition, an air embolism or infection can occur due to operator error [1-3]. Malfunctioning central venous catheters are usually caused by catheter malpositioning, which can lead to secondary complications such as errors in central venous pressure monitoring, erosion or perforations at vessel walls, hydrothorax, hydrops, cardiac tamponade, and edema and tissue damage at the catheter site [4].

The authors of this paper inserted a central venous catheter in a patient requiring aortic valve replacement through the right internal jugular vein and found the catheter misplaced into the right subclavian vein after performing a portable X-ray. Under

(c) This is an open-access article distributed under the terms of the Creative Commons Attribution Non-Commercial License (http://creativecommons.org/ licenses/by-nc/4.0/), which permits unrestricted non-commercial use, distribution, and reproduction in any medium, provided the original work is properly cited. 
image guidance by a mobile-type diagnostic X-ray apparatus (Carm fluoroscope), the authors were able to correct the position of the central venous catheter. We report the case herein.

\section{Case Report}

A 73-year-old female was hospitalized for aortic valve replacement to fix severe aortic regurgitation and stenosis. She had been subject to follow-up after being diagnosed with hypertension, aortic regurgitation, and stenosis five years earlier. During the follow-up, surgery was decided due to worsening symptoms and echocardiographic diagnosis. She was diagnosed with early gastric cancer in the antrum and was scheduled to undergo gastrectomy after recovery from heart surgery. Standing $144 \mathrm{~cm}$ tall and weighing $50 \mathrm{~kg}$, the patient showed vital signs within normal ranges except for signs of anemia, with a hemoglobin level of $8.5 \mathrm{mg} / \mathrm{dl}$ and hematocrit at $25.4 \%$

Prior to anesthetic induction, she was administered $2 \mathrm{mg}$ of midazolam by intravenous injection for sedation. Preoxygenation was performed through nasal cannula at $3 \mathrm{~L} / \mathrm{min}$. The right radial artery was secured, and invasive arterial pressure monitoring was begun. Prior to induction, the patient showed stable vital signs: an arterial blood pressure of $138 / 70 \mathrm{mmHg}$, a pulse of 66 beats/min, a breathing rate of 18 breaths/min, and $100 \%$ oxygen saturation of the peripheral vein. After administering $3 \mathrm{mg}$ of midazolam additionally, $2 \%$ propofol was infused using a target-controlled infusion pump (TCI pump) with a target plasma concentration of $3.0 \mu \mathrm{g} / \mathrm{ml}$. At the same time, induction was performed by administering $500 \mu \mathrm{g}$ of fentanyl diluted to $5 \mu \mathrm{g} / \mathrm{ml}$ in the burette infusion set by drip infusion. After allowing muscle relaxation with $10 \mathrm{mg}$ of vecuronium and checking for stabilization of the patient's vital signs, intubation was performed followed by mechanical ventilation with $\mathrm{O}_{2}$-medical air (fraction of inspired oxygen at 0.5 ).

In order to expand the internal jugular vein for central venous catheter insertion, the patient had her right arm fixed to the trunk in the Trendelenburg position. With a small pillow placed underneath the right shoulder and head turned to the left, the position facilitated access to the right internal jugular vein. A venipuncture site was sterilized for an aseptic procedure. Left-handed palpation was performed on the internal carotid artery, lying between and beneath two bodies of the right sternocleidomastoid muscle and located $3 \mathrm{~cm}$ above the superior border of the clavicle. An 18-gauge central venous needle was inserted towards the ipsilateral nipple at a point $1.5 \mathrm{~cm}$ medial to the external jugular vein. A $5 \mathrm{ml}$ syringe connected to the inserted needle was used for aspiration, and the color and pressure of the blood confirmed that it was venous blood. After removal of the syringe, a J-tip guide wire was inserted with its end pointing toward the heart. The guide wire was inserted smoothly up to $20 \mathrm{~cm}$. After removal of the needle and the incision on the skin, a 9-Fr introducer sheath (Advanced Venous Access $\mathrm{HF}^{\circledR}$, Edwards Lifesciences, Irvine, USA) accompanying dilator for the catheter was pushed in along the guide wire. There was a slight feeling of something getting stuck in the middle, which is normally experienced during successful central venous catheter placement. The insertion was made without much resistance, and the aspiration of blood from all three lumens of the catheter was successful. An intravenous catheter (PreSep ${ }^{\mathrm{TM}}$-central venous oximetry catheter set, Edwards Lifesciences, Irvine, USA) with each of its lumens filled with saline solution was inserted up to $20 \mathrm{~cm}$ through the introducer. Then, it was pulled back 3 $\mathrm{cm}$ to check again for blood aspiration. No noticeable resistance, arrhythmia, or changes in vital signs were observed during the insertion of the intravenous catheter. Monitoring of central venous pressure or central vein oxygen saturation was yet to be performed, and there were no signs of abnormal placement of the central venous catheter or other complications related to the procedure. However, a portable X-ray performed immediately after the procedure revealed that the end of the catheter was inserted to the ipsilateral subclavian vein (Figs. 1 and 2A). The authors first considered removing the inserted catheter entirely and using the left internal jugular vein but finally decided to correct the placement under image guidance with the catheter inserted after taking various circumstances into account.

In order to correct the placement of the catheter, the inserted intravenous catheter was removed. The J-tip guide wire was carefully inserted, once again, about $15 \mathrm{~cm}$ through the catheter introducer. The end of the catheter was pulled back to the internal jugular vein (Fig. 2B) and temporarily positioned. While a

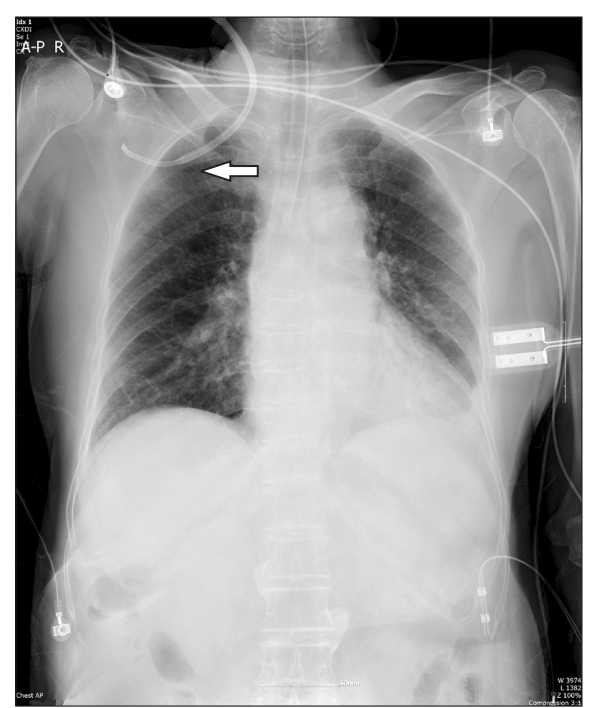

Fig. 1. Malposition of central venous catheter. Central venous catheter ( 9 Fr.) and central venous oximetry catheter $\left(\right.$ PreSep $^{\text {TM }}$ ) located in the right subclavian vein inserted via right jugular vein. 

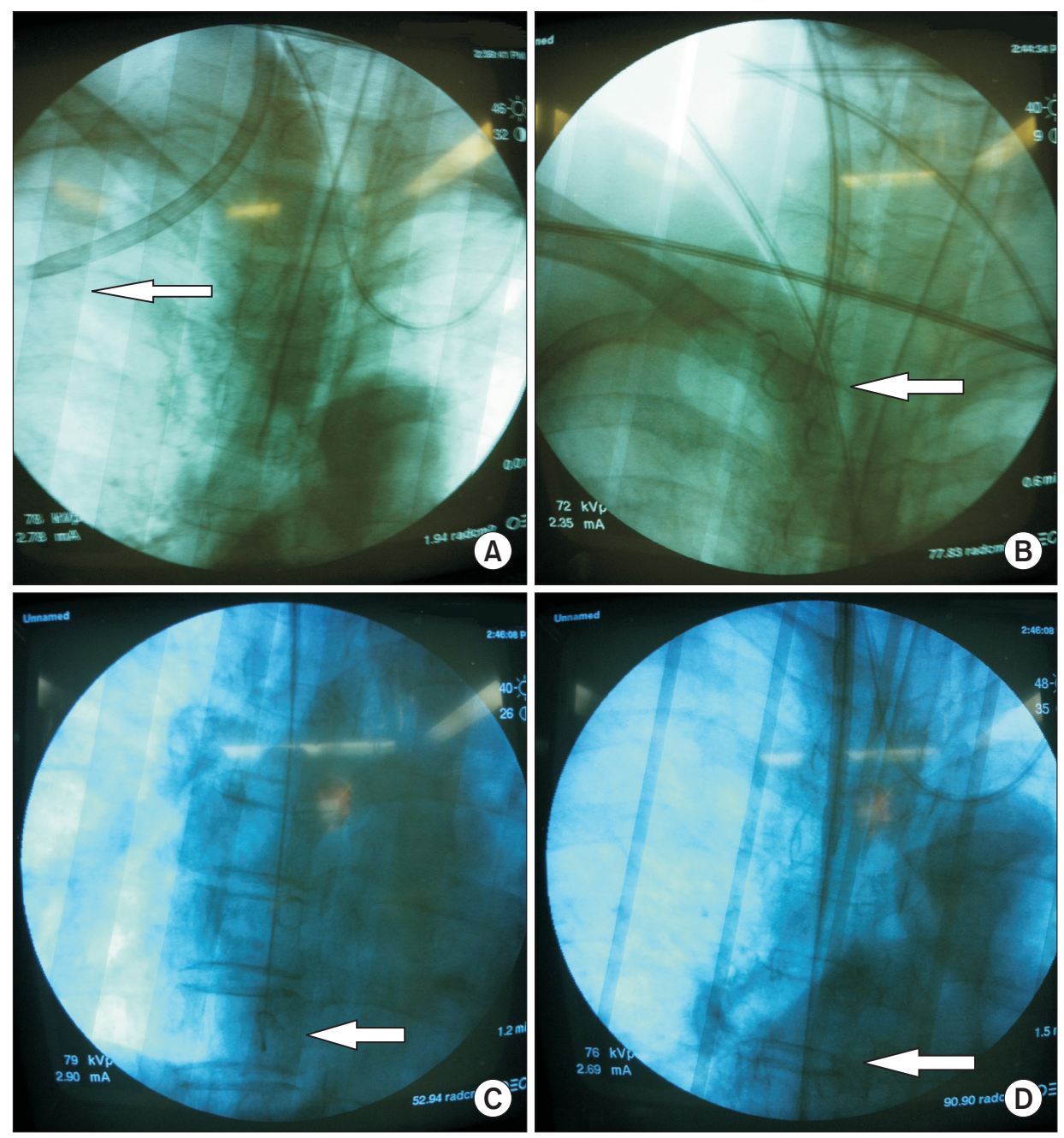

Fig. 2. Correction of the malposition of central venous catheter assisted by mobile type diagnostic X-ray apparatus ( $\mathrm{C}$-arm fluoroscope). The central venous catheter tips are located (A) in the right subclavian vein and (D) in the superior vena cava. The J-wire tips are located (B) in the junction of the right internal jugular vein and (C) in superior vena cava.

continuous X-ray was performed using a mobile-type diagnostic $\mathrm{X}$-ray apparatus (C-arm fluoroscope), the end of the J-tip guide wire was pulled back to the superior clavicle. Then, the J-tip was rotated $180^{\circ}$ towards inside and carefully inserted to the superior vena cava (Fig. 2C). This was followed by a successful attempt to position the end of the catheter near the junction of the right atrium along the corrected guide wire (Figs. 2D and 3). The entire process from catheter insertion to correction was executed aseptically under single venipuncture. The time spent correcting the malpositioning was less than 10 minutes.

No abnormal signs such as edema or hematoma were found on the patient's right shoulder and arm until the surgical procedure came to an end and she left the room. The patient had her central venous catheter removed one week later and was discharged from the hospital after receiving subtotal gastrectomy in the surgical department one month later.

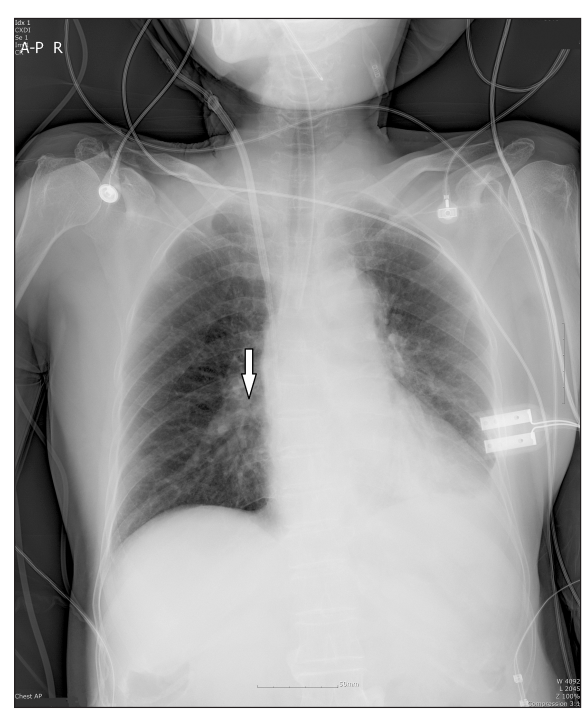

Fig. 3. Corrected position of central venous catheter ( 9 Fr.) and central venous oximetry catheter (PreSep ${ }^{\mathrm{TM}}$ ) in the junction of superior vena cava and right atrium. 


\section{Discussion}

A central venous catheter is usually placed into the internal jugular vein, subclavian vein, or femoral vein. The internal jugular vein has a relatively high risk of arterial puncture but a low risk of pneumothorax. The subclavian vein is relatively easy to access but runs a higher risk of pneumothorax and of malpositioning compared to the internal jugular vein. The femoral vein is rarely used; although it has an extremely low chance of malpositioning, it is highly likely to lead to thrombosis and infection [1-3].

The reported frequency of misplacement during central venous catheter insertion without image guidance ranges from $3.3 \%$ [5] to $6.2 \%$ [6]. The main factors affecting catheter malpositioning are stenosis or occlusion in the anatomical structure of a blood vessel. Pikwer et al. [5] reported frequencies of malpositioning of $1.4,3.8,9.1$, and $2.7 \%$ when using the right internal jugular vein, left internal jugular vein, right subclavian vein, and left subclavian vein, respectively. Schummer et al. [6], meanwhile, reported frequencies of $4.3,12.0,9.3$, and $8.4 \%$ for the right internal jugular vein, left internal jugular vein, right subclavian vein, and left subclavian vein, respectively. The insertion of a central venous catheter through the right internal jugular vein is reported to have the lowest chance of malpositioning because, anatomically, the blood vessel runs without much winding towards superior vena cava. When the right subclavian vein or left internal jugular vein were used, however, cases of malpositioning were reported in the superior intercostal vein, the azygos vein [7], the anomalous pulmonary vein [8], and the internal mammary vein [9], as the blood vessel in these locations is diverted into several smaller vessels.

Muhm et al. [10] and Singh et al. [11] reported that a major factor leading to central venous catheter malpositioning is the catheter type. A soft silicon catheter is said to be more likely to travel in an unintended direction and result in a higher frequency of malpositioning than a semi-rigid catheter.

Pikwer et al. [5] found that the patient's age and gender, the difficulty of the catheterization, the number of trials, the period of catheter insertion, and surgical skills did not have a significant impact on central venous catheter misplacement. Instead, they highlighted the type of blood vessel selected and its anatomical changes as the most important factor.

Located under the sternocleidomastoid muscle, the internal jugular vein forms an acute angle with the subclavian vein towards the heart, together forming the brachiocephalic vein. In particular, the right internal jugular vein runs almost straight until it reaches the right atrium, passing the brachiocephalic veins and superior vena cava. Therefore, it has the lowest risk of malpositioning during central venous catheter insertion. The subclavian vein runs almost parallel behind the clavicle, com- bining with the internal jugular vein laterally in a horizontal direction. The internal jugular vein is commonly found adjacent and antero-lateral to the carotid artery. In some cases, however, it may be located in front of the carotid artery or $1 \mathrm{~cm}$ away from it $[12,13]$.

The authors inserted a central venous catheter through the right internal jugular vein, which has the lowest frequency of malpositioning, and used a 9 Fr semi-rigid catheter accompanying dilator. Nonetheless, there occurred misplacement into the right subclavian vein, which is not easily accessible without much resistance as it forms an acute angle with the internal jugular vein. This can be attributed to the patient's anatomical structure; although it was not measured, the angle between the patient's internal jugular vein and subclavian vein on the same side was larger than that of a normal person. Other possible causes for the access to the subclavian vein are venous distension from the Trendelenburg position, movement of the internal jugular vein due to the displacement of the head to the left, and adduction of the right arm. This position, however, is necessary to identify surface anatomical landmarks for venipuncture and facilitate insertion. We do not consider there to be any need to shun this practice only in order to prevent the type of rare complication observed in this case.

Other possibilities include anatomical variations in the internal jugular vein, such as duplication or fenestration causing the diverted internal jugular vein towards the outside to meet the subclavian vein. Prades et al. [14] witnessed an anomaly of the internal jugular vein in roughly $0.4 \%$ of neck dissections and reported lateral division of the accessory nerve passing between duplicated internal jugular veins in all cases. In addition, Micozkadioglu and Erkan [15] reported cases of neck dissection where phlebectasia of the right internal jugular vein took place, with its division moving laterally.

However, even experienced physicians find it challenging to predict anatomical variations in patients and run the risk of misplacing catheters into the subclavian vein or the internal jugular vein on the opposite side. Resistance during central venous catheter insertion, poor blood aspiration after insertion, excessively high CVP, and abnormal waveform may all be obvious signs of catheter malpositioning. However, variations in a patient's anatomical structure are often hard to detect. Therefore, a test should be performed using ultrasound or diagnostic X-ray after central venous catheter insertion in order to rule out and prevent complications, including malpositioning. Even in the event of anatomical variations, such image-guided assistance will help correct the misplacement.

The National Institute for Health and Clinical Excellence (NICE) Guidelines suggest ultrasound-guided central venous catheter insertion. This technique avoids complications such as arteriopuncture, pneumothorax, and multiple venipuncture due 
to difficulties locating the proper blood vessel, thereby increasing the chance of successful procedure. In addition, X-ray helps in correcting the malpositioning and determining the length of the central venous catheter or the exact location of the catheter tip. Ultrasound is useful in identifying the target blood vessel and needle insertion site, while X-ray is believed to be more effective in checking the exact location of the guide wire and catheter tip and in watching the continuous insertion process. This may be partly explained by the fact that clearly identifying structures with ultrasound requires more expertise than with X-ray.

The authors generally use surface anatomical landmarks for central venous catheter insertion and therefore, for this case, did not use image-guided techniques, such as ultrasound, from the initial insertion. Fortunately, however, venipuncture of the internal jugular vein was successful at the first attempt. After locating the catheter tip using a portable X-ray and realizing it was wrongfully inserted into the ipsilateral subclavian vein, the authors had to correct the position of the catheter tip instead of locating the internal jugular vein. A portable X-ray was used, as no ultrasound device was readily available in the operating room. Another attempt at insertion involving the removal of the catheter might have been expensive and time-consuming and may have increased the chance of complications due to repeated venipuncture. An image-guided central venous catheter insertion technique improves the chance of correct placement and reduces the chance of complications. Therefore, a growing number of cases use an image-guided technique from the initial stage considering the time and money spent on unwanted multivenipuncture or complications arising from failed catheter insertion.

\section{References}

1. Paoletti F, Ripani U, Antonelli M, Nicoletta G. Central venous catheters. Observations on the implantation technique and its complications. Minerva Anestesiol 2005; 71: 555-60.

2. Wicky S, Meuwly, Doenz F, Uske A, Schnyder P, Denys A. Lifethreatening vascular complications after central venous catheter placement. Eur Radiol 2002; 12: 901-7.

3. Polderman KH, Girbes AJ. Central venous catheter use Part 1: mechanical complications. Intensive Care Med 2002; 28: 1-17.

4. Boardman P, Hughes JP. Radiological evaluation and management of malfunctioning central venous catheters. Clin Radiol 1998; 53: 10-6.

5. Pikwer A, Bååth L, Davidson B, Perstoft I, Akeson J. The incidence and risk of central venous catheter malpositioning: a prospective cohort study in 1619 patients. Anaesth Intensive Care 2008; 36: 30-7.

6. Schummer W, Schummer C, Rose N, Niesen WD, Sakka SG. Mechanical complications and malpositions of central venous cannulations by experienced operators. A prospective study of 1794 catheterizations in critically ill patients. Intensive Care Med 2007; 33: 1055-9.

7. Currarino G. Migration of jugular or subclavian venous catheters into inferior tributaries of the brachiocephalic veins or into the azygos vein, with possible complications. Pediatr Radiol 1996; 26: 439-49.

8. Townley SA. Central venous catheter malposition in an anomalous pulmonary vein. Eur J Anaesthesiol 2003; 20: 985-6.

9. Sandroni C, Pirronti T, Tortora F, Santangelo S, Rinaldi P, Antonelli M. Unusual central venous catheter malposition into the left internal mammary vein: a case report. Intensive Care Med 2003; 29: 2338-9.

10. Muhm M, Sunder-Plassmann G, Apsner R, Pernerstorfer T, Rajek A, Lassnigg A, at al. Malposition of central venous catheters. Incidence, management and preventive practices. Wien Klin Wochenschr 1997; 109: 400-5.

11. Singh PK, Ali Z, Rath GP, Prabhakar H. Catheter malposition following supraclavicular approach for subclavian vein catheterisation-case reports. Middle East J Anesthesiol 2008; 19: 1405-10.

12. Kadir S. Atlas of normal and variant angiographic anatomy. Philadelphia, Saunders. 1991, pp 203-25.

13. Parviz J, Joseph BW Jr, Robert JG, Richard LF, William WM, Nathalie A, et al. Surgical Anatomy of the Head and Neck. Philadelphia, Lippincott Williams \& Wilkins. 2001, pp 707-10.

14. Prades JM, Timoshenko A, Dumollard JM, Durand M, Merzougui N, Martin C. High duplication of the internal jugular vein: clinical incidence in the adult and surgical consequences, a report of three clinical cases. Surg Radiol Anat 2002; 24: 129-32.

15. Micozkadioglu SD, Erkan AN. Internal jugular vein anomaly: a lateral branch of the internal jugular vein in the neck. EJENTAS 2011; 12: 77-9. 